\title{
Study on the Influence of Pharmaceutical Management on Rational Drug Use under Chinese Health Strategy
}

\author{
Lijuan Cheng \\ Yichun Vocational Technology College, Yichun 336000, China \\ 20175490@qq.com
}

\begin{abstract}
In order to better promote the implementation of the healthy China strategy and the innovation of public hospital system management, we can explore a modern public hospital management system that can meet the needs of the people in the new era from the aspects of medical service supply mode, collaborative governance design of public hospitals and internal management system of public hospitals. In this paper, 300 prescriptions in our hospital before and after May, 2020 were selected as research objects, and were randomly divided into two groups, one group was the control group (300 cases) without pharmaceutical management, and the other group was the observation group (300 cases) with pharmaceutical management. Then, the drug satisfaction, irrational drug use and inappropriate drug use of the two groups were compared. The results showed that the satisfaction of medication in the observation group was significantly better than that in the control group, and the irrational and inappropriate medication was lower than that in the control group, with statistical differences $(P<0.05)$. It shows that the implementation of pharmaceutical management measures under the strategy of Healthy China can effectively improve patients' satisfaction with medication and prevent irrational medication.
\end{abstract}

Keywords: Health Strategy in China; Pharmaceutical Management; Rational Drug Use; Influence.

\section{Introduction}

Pharmaceutical management is one of the important components of the hospital. Outpatient medicine directly faces patients, which is the representative of the mental outlook of the hospital and the reflection of the overall image of the hospital [1]. The quality of pharmaceutical management is closely related to the reputation and development of hospitals. Rational drug use can quickly relieve patients' illness and is of great significance to patients' rehabilitation. In recent years, the irrational clinical drug use in China is on the rise, which not only threatens the life safety of patients, but also easily leads to disputes between doctors and patients, which affects the reputation of hospitals and is not conducive to the stable development of hospitals [2-3]. The implementation of pharmaceutical management can effectively improve this problem. By analyzing the requirements of the strategy of healthy China in the new era, this paper explores the influence of pharmaceutical management on rational drug use from three aspects: medical service supply mode, public hospital governance system and internal management system of public hospitals, to provide reference for the innovation of rational drug use in hospitals.

\section{Connotation and General Requirements of China's Health Strategy}

By interpreting the overall framework and logical relationship of the healthy China strategy, we think that it contains a basic value and three basic practical strategies [4]. The basic value is "Peoplecentered". Around this center, the healthy China strategy requires that a healthy country be built in an All-round way and the people's health level be improved from three practical strategies: "building an All-round and Full-cycle health service system", "deepening the reform of the medical and health system" and "building and sharing health policies to integrate people with all policies". All the above three dimensions put forward corresponding specific requirements for the reform and innovation of public hospitals (as shown in Figure 1). 


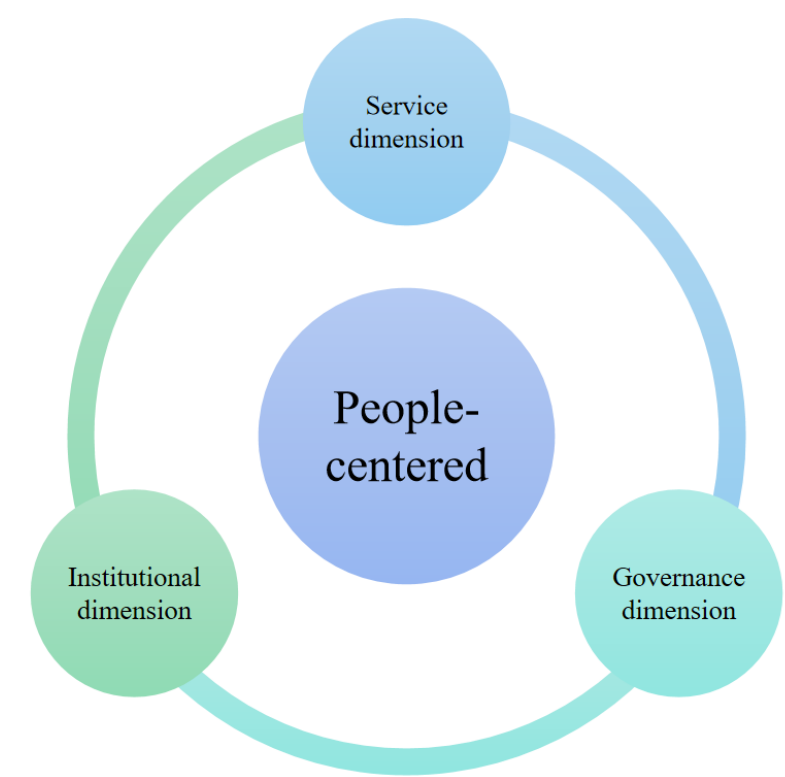

Figure 1. Logical framework of China's health strategy

\subsection{People-centered}

In the new era, the people's growing health needs will not always stay at the stage of "getting medical treatment for the sick". Public hospitals are the window for people to contact with national health services, and need to change from pure treatment orientation to paying attention to "patient service" in a more complete sense. They should not only provide high-quality diagnosis and treatment services, but also pay attention to patients' experiences, such as improving medical procedures to improve convenience, improving the humanistic environment for medical treatment, respecting patients' privacy, safeguarding patients' dignity, ensuring information sharing and protecting patients' right to know.

\subsection{Provide All-round and Full-cycle Health Services}

China's health strategy requires providing people with "All-round and Full-cycle health services". Its core essence lies in: first, shifting the focus of work from disease treatment to prevention and control of health risk factors; Second, focusing on people's various health needs, we will provide health services covering prevention, diagnosis and treatment of acute/chronic diseases, rehabilitation nursing, old-age care and other systems. Under the guidance of this goal, the mode of providing medical services and the management system of medical institutions is in urgent need of reform and innovation.

\subsection{Deepen the Reform of the Medical and Health System}

How to transform from the traditional administrative bureaucratic management mode to the modern new governance mode is another key requirement for the management of public hospitals to implement the healthy China strategy [5]. This requires not only the courage and wisdom of government departments, but also the continuous self-improvement and innovation of public hospitals.

\subsection{Co-construction and Sharing}

Under the guidance of this governance concept, the idea of multi-sectoral linkage of health management and source governance will replace the traditional idea that a single business department only aims at the terminal control of disease treatment. This requires that public hospitals, as the traditional providers of medical services, cooperate with other relevant subjects, and at the same time, change their working policies from the traditional disease-oriented to the people's health, which involves a profound change in incentives and behaviors of public hospitals. 


\section{Research Method}

\subsection{General Information}

After May 2020, 300 patients were selected as the observation group, including 163 males and 137 females, aged from 15 to 65 years, with an average of (46 \pm 2$)$ years. Meanwhile, 300 patients before May 2020 were selected as the control group, including 155 males and 145 females, aged from 15 to 65 years, with an average age of $(46 \pm 2)$ years. All the selected cases in the two groups met the clinical medication indications [6], and the patients themselves informed and agreed to the medication.

\subsection{Inclusion and Exclusion Methods}

Inclusion criteria: (1) All patients knew and agreed to this study, and all of them signed informed consent; (2) The patients participated in the study all the way, and never quit.

Exclusion criteria: (1) exclude patients with mental disorders or mental illness; (2) Severe patients with liver and heart were excluded.

\subsection{Method}

The control group was carried out according to the routine system of hospital outpatient pharmacy, that is, taking medicines for patients according to their prescriptions, and informing them of the ways of taking medicines.

The observation group carried out pharmaceutical management by the following methods:

(1) Establish a drug management team: a pharmacist, a doctor and three other drug-related personnel will form a drug management team to formulate the standards for rational drug use, and manage and track the drug use.

(2) Improvement of drug management system: Based on existing drugs, combining with clinical problems and experiences, the formulation of drug management is completed, including prescription process system, classified management system and so on.

(3) Increase the supervision of pharmacists' work: strengthen the supervision of pharmacists' work, do a good job in the allocation and supervision of pharmacists, ensure the accuracy of medication information, train and learn pharmacists regularly, enrich pharmacists' professional knowledge, and avoid the occurrence of irrational medication events such as adequate medication and measures of administration due to the renewal of drugs.

(4) The adverse reactions caused by drugs should be counted and reported to the drug monitoring center in time, and a case data center should be established, to accurately grasp the information of patients. Nursing staff should actively communicate with patients, understand the actual medication situation of patients and the problems existing in the medication process, and try to reduce the incidence of adverse reactions caused by irregular drug use as much as possible.

\subsection{Evaluating Indicator}

The scores of satisfactions with prescription medication were compared between the two groups, and the incidents of irrational prescription medication and unsuitability of prescription medication between the two groups were compared, and detailed statistics and analysis were made. Among them, the full score of medication satisfaction is 100 points, 80 points above are satisfactory, and 80 points below are unsatisfactory. It mainly evaluates the awareness of prescription medication, medication guidance and medication effect.

\subsection{Statistical Analysis}

The statistics were analyzed by SPSS 19.0, and the measurement data were expressed by (mean standard \pm deviation). If $\mathrm{p}<0.05$, the data has statistical significance. 


\section{Result}

\subsection{Satisfaction Comparison}

The drug satisfaction of the observation group with pharmaceutical care was $93.7 \%$, while that of the control group without pharmaceutical care was 51.3\%. The comparison showed that the drug satisfaction of the observation group was significantly better than that of the control group, with a statistically significant difference $(\mathrm{P}<0.05)$, as shown in Figure 2 below.

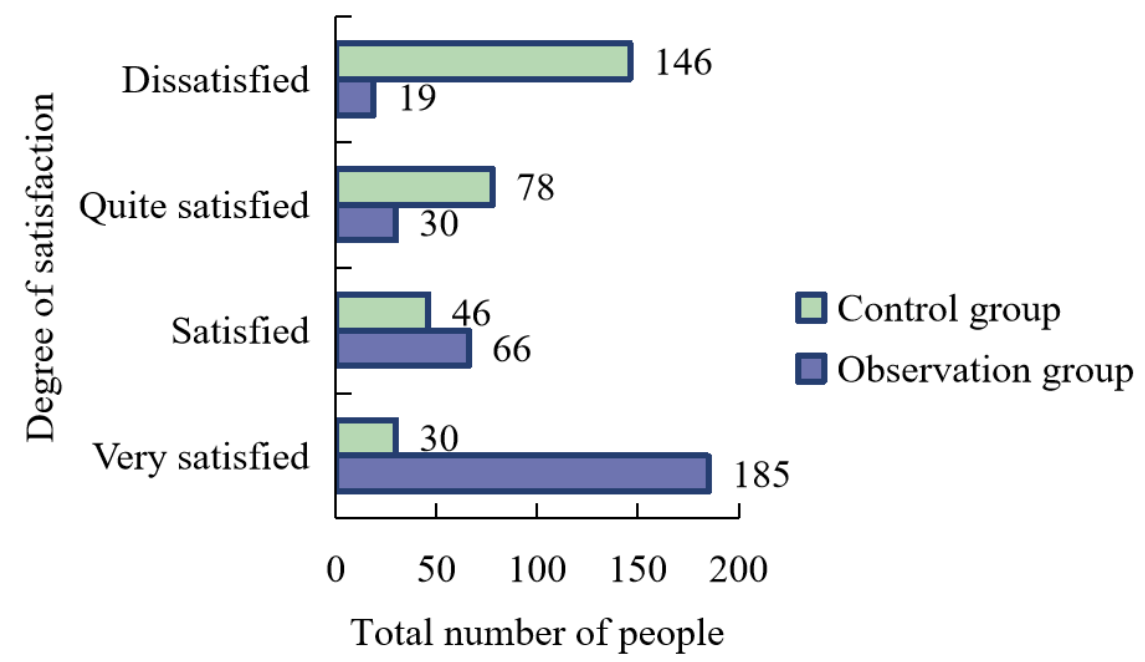

Figure 2. Comparison of drug satisfaction between two groups of patients

\subsection{Comparison of the Incidence of Irrational Prescription Medication between the Two Groups}

The irrational rate of prescription medication in the observation group was $8.7 \%$, which was lower than that in the control group $(23.0 \%)$, with significant statistical difference $(\chi 2=35.039, \mathrm{P}<0.05)$, as shown in Table 1.

Table 1. Incidence of irrational prescription among groups (n, \%)

\begin{tabular}{|c|c|c|c|c|c|}
\hline Group & $\begin{array}{c}\text { Number of } \\
\text { cases }\end{array}$ & $\begin{array}{c}\text { Repeated } \\
\text { medication }\end{array}$ & $\begin{array}{c}\text { Wrong dosage and } \\
\text { dosage form }\end{array}$ & $\begin{array}{c}\text { Wrong way of } \\
\text { administration }\end{array}$ & $\begin{array}{c}\text { Incidence } \\
\text { rate }\end{array}$ \\
\hline $\begin{array}{c}\text { Observation } \\
\text { group }\end{array}$ & 300 & $12(4.0)$ & $25(8.3)$ & $32(10.7)$ & $69(23.0)$ \\
\hline Control group & 300 & $5(1.7)$ & $8(2.7)$ & $13(4.3)$ & $26(8.7)$ \\
\hline$\chi^{2}$ & & & & & 35.039 \\
\hline $\mathrm{P}$ & & & & & 0.003 \\
\hline
\end{tabular}

\section{Discussion}

In recent years, the problem of difficult and expensive medical treatment has become increasingly serious, which leads to the increasingly tense doctor-patient relationship. If the hospital can't provide high-quality nursing service, it will probably lead to disputes between doctors and patients. In order to improve the doctor-patient relationship, reducing the medical cost is the most critical. If professionals can participate in clinical treatment from prescriptions, it can effectively reduce drug abuse, improve the rationality of drug use and reduce the cost of drug use for patients. As an important component of the hospital, pharmacy is an important subject of the hospital, and the quality of pharmaceutical management has a very important impact on the safe medication of clinical patients. Therefore, strengthening drug management in clinical practice to ensure the standardization and accuracy of drug use will play a significant role in promoting the safety of drug use and the healthy development of medical and health undertakings. 
Drug use is one of the inevitable clinical problems. The occurrence of irrational drug use not only damages the health of patients, but also damages their trust in medical treatment, which is not conducive to the formation of a good doctor-patient relationship. In this experiment, after strengthening pharmaceutical management in our hospital, the incidence of irrational drug use decreased greatly, and the problems that patients often feedback were unclear and incomprehensible were improved, and patient satisfaction was also greatly improved. This shows that pharmaceutical management does have its advantages. It can strengthen the management of drugs, doctors and pharmacists through scientific and rigorous methods, which is helpful to reduce the occurrence of irrational drug use and improve the quality of prescriptions.

Pharmaceutical management takes patients as the center, which directly reflects the working confidence and attitude of relevant personnel. Only by analyzing the rational drug use from the perspectives of patients and hospitals themselves can we truly highlight the practical value of rational drug use.

For patients, rational drug use requires providing them with the best drug effect, the best performance and the lowest cost, so as to give consideration to the safety, effectiveness and economy of drug treatment.

For hospitals, systematic pharmaceutical management can promote rational drug use, and after rational drug use, the current medical situation in hospitals will be changed. It plays a significant role in improving the quality of medical services, perfecting the system construction and improving the quality of patients' diagnosis and treatment. The results showed that the patients in the observation group scored higher than those in the control group, and the incidence of irrational prescription drugs and inappropriate prescription drugs were lower than those in the control group $(\mathrm{P}<0.05)$.

Clinical treatment is the core of hospital and the most complex part of hospital services, which is due to its own great difficulty, high heterogeneity of patients, and deviation due to incomplete and inaccurate information provided by patients or different interpretations of test results and patients' symptoms by different medical staff. However, it is a common phenomenon in hospitals in China that more attention is paid to various examination techniques, while less attention is paid to the treatment methods formulated spontaneously for patients and less attention is paid to patients' psychological feelings. The purpose of setting up the patient management and innovation center is to make the hospital meet the needs of the people in the new era with clear objectives and in an organized way, so that patients can participate in medical treatment and health management, thus achieving a winwin situation between the hospital and patients and helping to improve the doctor-patient relationship.

As a training material for medical staff. In order to promote the innovation of doctor-patient cooperation, it is suggested to add three departments:

(1) Technology collaborative innovation department is responsible for organizing and coordinating innovative cooperation methods, integrating innovative technologies, actively seeking innovative transformation methods, and regularly reporting innovative achievements to other centers.

(2) The management department of difficult diseases regularly publishes the research progress of difficult diseases concerned by the hospital to patients, and encourages patients to participate in medical research, including organizing clinical trials and seeking alternatives, and putting forward suggestions on methods to cure themselves or their loved ones, etc. The hospital can provide support, help evaluate and participate in discussions.

(3) The big data management department is mainly positioned to integrate the process of patients' self-management of chronic diseases and explore ideas and methods to reduce costs. An open data platform can be designed, and patients can regularly upload and update the data of symptom development, medication effect and rehabilitation effect of related diseases. Any innovation that can improve health and reduce medical expenses is very much needed by patients, so that they are more motivated to participate in the innovation of medical technology and health management, and regularly analyze and integrate the data to provide data basis for innovation. These innovations depend on the development of information technology [7]. 


\section{Summary}

To sum up, the development of pharmaceutical management under the healthy China strategy has significantly reduced the occurrence of irrational drug use in clinic, and is conducive to the improvement of doctor-patient relationship. Patients have a high degree of recognition of this management measure, which is worthy of wide application in clinic.

\section{References}

[1] Chen Yuanyuan. the influence of pharmaceutical management on rational drug use [J]. Chinese community physicians, 2020, v.36; No.809(11):10+12.

[2] Liu Jianping. Study on the influence of pharmaceutical management on rational drug use [J]. Electronic Journal of Clinical Medical Literature, 2020, v.7; No.442(21):198-198.

[3] Yu Fengjuan. Analysis of the influence of pharmaceutical intervention management on rational drug use in county hospitals [J]. Journal of Psychology, 2019, 014(004):111-112.

[4] Chen Zhuo. Analysis of the influence of pharmaceutical management on rational drug use [J]. Health Care Guide, 2019, 000(017):200.

[5] Liu Jinghua, Tian Yu. Analysis of the influence of pharmaceutical management on rational drug use [J]. Science and Health Care, 2019, 022(010):293-294.

[6] Liu Qiaoyu. The influence of pharmaceutical management on rational drug use [J]. Health Care Guide, 2019, 000(007):192.

[7] $\mathrm{Xu}$ xuechao. analysis of the influence of hospital pharmacy intervention management on standardizing outpatient prescriptions and rational drug use [J]. required health reading, 2019, 000(024):290-291. 